\title{
A multi-scale, multi-domain approach for LES of high Reynolds number wall-bounded turbulent flows
}

\author{
R. Akhavan and M. U. Haliloglu \\ Department of Mechanical Engineering, University of Michigan, \\ Ann Arbor, MI 48109-2125, USA
}

A major impediment to LES of high Reynolds number wall-bounded turbulent flows is the prohibitive resolution requirements of LES in the near-wall region. In the recent years, a number of wall modelling approaches have been proposed to bypass these resolution requirements (see [1] for a detailed review). Existing approaches are generally based on solving a reduced set of RANS equations in the near-wall region to obtain appropriate boundary conditions for LES, which is applied only in the outer layer of the flow. In application, these approaches have generally proved to be wanting in accuracy or scope [1].

In this study, we present a new multi-domain, multi-scale approach for LES of high Reynolds number wall-bounded turbulence. This approach capitalizes on the well-known quasi-periodicity of the turbulence structures in the near-wall region to solve the near-wall region in a minimal flow domain. This near-wall domain is then repeated periodically (or quasi-periodically) and matched to a full-domain LES in the core, solved at a much coarser resolution. This multi-scale approach has been implemented in LES of turbulent channel flow using a non-conforming, spectral domaindecomposition (patching) method. A schematic of the channel and the domains used in the nearwall and outer regions is shown in Fig. 1. Simulations have been performed for $R e_{\tau} \sim 1000$, 2000, 5000, 10,000, and 20,000. The subgrid-scale model used in the simulations is the Nonlinear Interactions Approximations (NIA) model, recently developed in our group [2]. This model uses graded filters and deconvolution to directly model the nonlinear terms in the filtered Navier-Stokes equations, instead of the traditional subgrid-scale stresses. The NIA model has been shown to give very accurate predictions of both the filtered and full (filtered plus subgrid) turbulence statistics. In the multi-scale approach, the near-wall region is solved in a domain spanning the full length of the computational domain in the streamwise $(x)$ direction, a height of $100-250$ wall units in the wall-normal $(z)$ direction, and a width of several hundred wall units in the spanwise $(y)$ direction. The near-wall domain is solved with typical resolutions of $\Delta x^{+} \sim(100-250)$ in the streamwise direction, $\Delta y^{+} \sim(25-50)$ in the spanwise direction, and $\Delta z^{+}<(15-35)$ in the wall-normal direction. In the core region, the LES equations are solved in the full domain, of size $L_{x}^{+}=L_{y}^{+}=\frac{\pi}{2} R e_{\tau}$, with typical resolutions of $\Delta x^{+} \sim \Delta y^{+} \sim(100-250)$ and $\Delta z^{+}<(100-500)$. Fig. 2 shows the results obtained using this multi-scale approach with a periodic repetition of the near-wall domain. Good agreement is observed between the predicted turbulence statistics and available experimental data. Studies are currently under way to determine the minimum/optimal size of the near-wall unit and ways for implementing more physical quasi-periodic repetition of the near-wall domain. The findings will be discussed.

\section{References}

[1] Piomelli, U. \& Balaras, E. 2002 Annu. Rev. Fluid Mech., 34, 349-74.

[2] Haliloglu, M.U. \& Akhavan, R. 2003 In Direct and Large-Eddy Simulation V, edited by R. Friedrich, B.J. Geurts, and O. Metais (Kluwer Academic Publishers). 


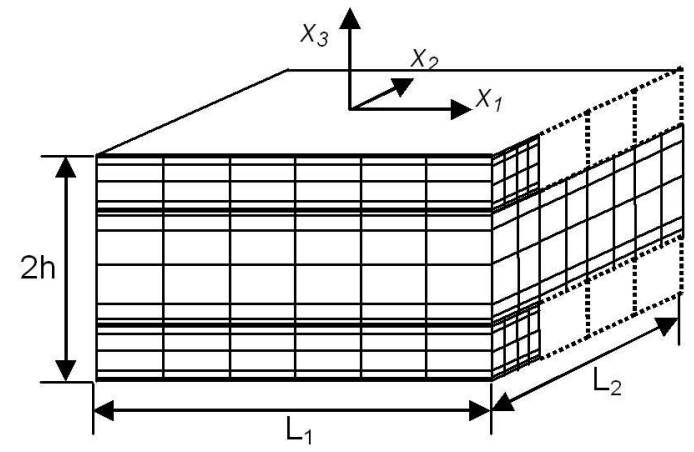

Figure 1: A schematic of the multi-domain channel.
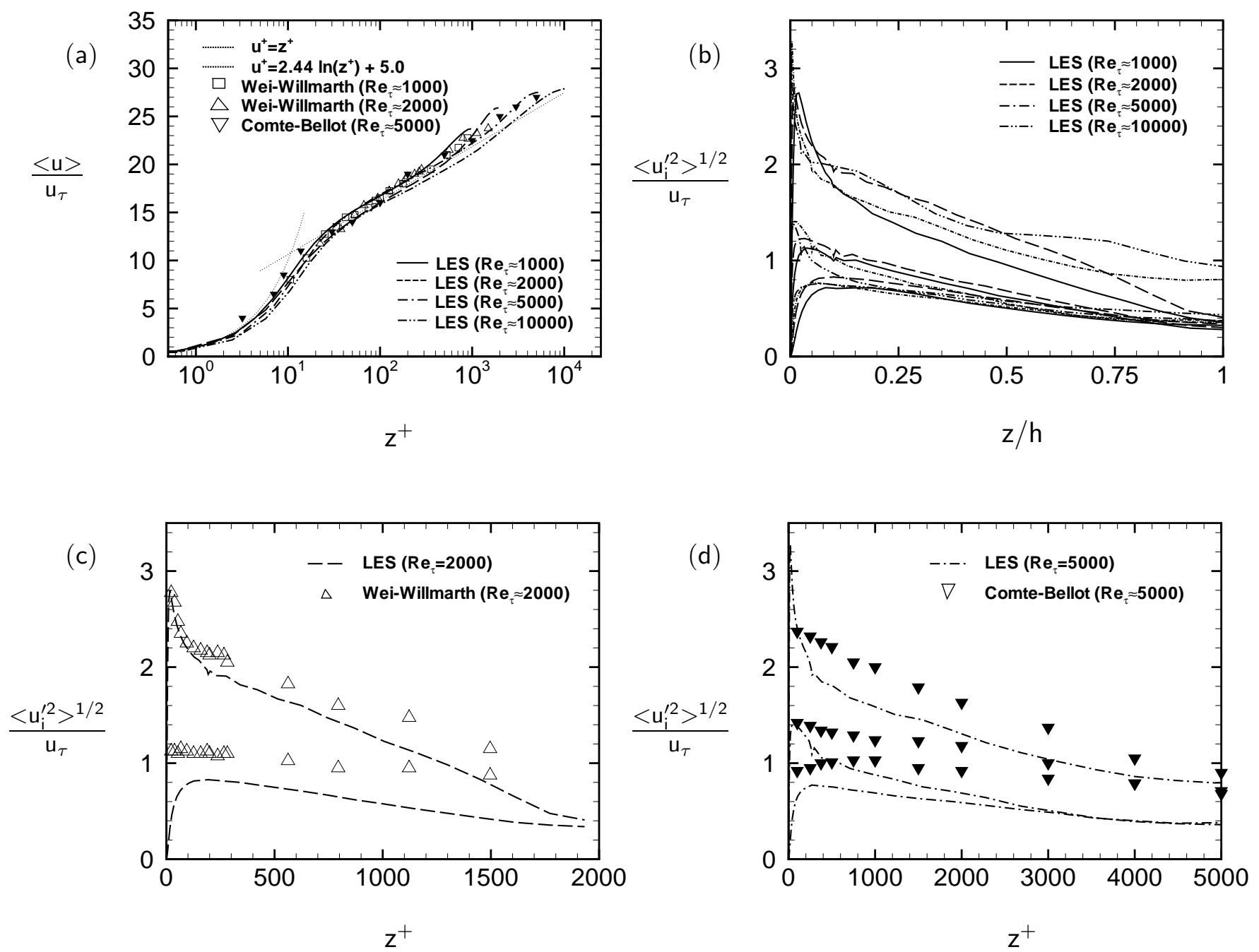

Figure 2: Turbulence statistics predicted by LES compared to available experimental data. 